\title{
On the structure of band edges of 2-dimensional periodic elliptic operators
}

by

NikOLAY FiLONOV

V. A. Steklov Mathematical Institute

St. Petersburg, Russia

and

St. Petersburg State University

St. Petersburg, Russia
ILYA KACHKOVSKIY

Michigan State University East Lansing, MI, U.S.A.

To the memory of Yuri Safarov, our dear friend and colleague.

\section{Introduction}

The structure of band edges of periodic Schrödinger operators is an interesting and wide open question of mathematical physics. For example, suppose that a band function $k \mapsto E(k)$ has a minimum (or maximum) $k_{0}$. In solid state physics, the tensor of effective masses $M_{\text {eff }}$ at $k_{0}$ is defined as

$$
\left\{M_{\mathrm{eff}}^{-1}\right\}_{i j}= \pm\left.\frac{1}{\hbar^{2}} \frac{\partial^{2} E}{\partial k_{i} \partial k_{j}}\right|_{k=k_{0}}
$$

(see [1, Chapter 12, equation (12.29)] for more details). The choice of the sign depends on whether the extremum is a minimum ("+", the effective mass of an electron) or a maximum ("-", the effective mass of a hole). This definition of $M_{\text {eff }}$ makes sense only if the right-hand side is invertible, i.e. if the critical point $k_{0}$ is non-degenerate. This is always true in one dimension; see, for example, [21, §XIII.16]. It is commonly believed that, for $d \geqslant 2$, the spectral gap edges are non-degenerate for "generic" potentials; see, for example, [18, Conjecture 5.1] and the recent review [15, §5.9.2]. However, there are very few rigorous results in this direction. In [11], it is shown that the lowest eigenvalue for the periodic Schrödinger operator is non-degenerate. The same holds for the 2dimensional Pauli operator; see [5]. A wide class of operators for which the lower edge

The first author was supported by RFBR Grant 16-01-00087 and by Simons Foundation. The second author was supported by AMS Simons Travel Grant 2014-2016 and by NSF grant DMS-1758326. 
of the spectrum can be extensively analysed is described in [6]. See also the survey [14] on photonic crystals, where additional references are given. For periodic magnetic Schrödinger operators, even the lowest eigenvalue may be degenerate (i.e. the righthand side of (1.1) may vanish; see [23]). Note, however, that this can happen only for sufficiently large magnetic potentials, as shown in [24].

Much less is known about the edges of other bands. In [12], it is established that, for periodic operators of the form $-\Delta+V$ with generic $V$, the edge of each spectral gap is an extremum of only one band function, but the question of non-degeneracy of these extrema remains open. In [25], it is shown in two dimensions that for any $N$ there exists a $C^{\infty}$-neighbourhood of zero such that, for potentials $V$ from a dense $G_{\delta}$-subset of that neighbourhood, the first $N$ band functions are Morse functions. In other words, any finite number of bands is non-degenerate for generic $C^{\infty}$-small potentials.

In the present paper, we establish the following result (Theorem 2.1): for a wide class of 2-dimensional periodic elliptic second-order operators, any global minimal or maximal value of any band function can only be attained at a discrete set of points. In other words, the global extrema of each band function are isolated. In particular, this implies that the level sets corresponding to spectral band edges cannot contain 1-dimensional curves. We do not need any genericity or smallness assumptions, and our result holds for all bands, not necessarily for the edges of the spectrum. We formulate the results for "smooth" second-order elliptic operators (2.1). We believe that, using methods from [22], the result can be extended to the same generality in which the absolute continuity of the spectrum in two dimensions is established. The extension beyond dimension 2 , however, seems significantly more challenging, as our technique relies heavily on 2-dimensional specifics.

An immediate consequence of our result is that Liouville theorems (in the sense of [17] and [18]) hold for the operator (2.1) at all gap edges; see Corollary 2.2. Our result can also be used in studying Green's function asymptotics near spectral gap edges (see [10], [19] and [9]) and to obtain a "variable period" version of the non-degeneracy conjecture in two dimensions [20].

Surprisingly, the statement of the main theorem fails for discrete periodic Schrödinger operators on $\mathbb{Z}^{2}$, already in the case of a diatomic lattice. We explain the corresponding example of non-isolated extrema in $\S 7$.

Acknowledgements. The results were partially obtained during the programme Periodic and Ergodic Spectral Problems in January-June 2015, supported by EPSRC Grant EP/K032208/1. We are grateful to the Isaac Newton Institute for Mathematical Sci- 
ences, Cambridge, for their support and hospitality. We would like to express our deepest thanks to Peter Kuchment, Leonid Parnovski, and Roman Shterenberg for several lively and fruitful discussions during the programme, and to Alexander Pushnitski for reading the draft version of the text and for valuable remarks. We are also grateful to the anonymous referee, whose suggestions significantly improved the quality of the paper and the references.

\section{Main result}

Let

$$
\Gamma=\left\{n_{1} b_{1}+n_{2} b_{2}: n_{1}, n_{2} \in \mathbb{Z}\right\}
$$

be a lattice in $\mathbb{R}^{2}$, and let $\Omega \subset \mathbb{R}^{2}$ be an elementary cell of $\Gamma$ identified with $\mathbb{R}^{2} / \Gamma$. We will use notation such as $\mathrm{C}_{\text {per }}^{1}(\Omega)$ and $H_{\text {per }}^{1}(\Omega)$ for the classes of functions satisfying periodic boundary conditions.

The periodic magnetic Schrödinger operator with metric $g$ is defined by the expression

$$
(H u)(x)=(-i \nabla-A(x))^{*} g(x)(-i \nabla-A(x)) u(x)+V(x) u(x),
$$

where the electric potential $V: \mathbb{R}^{2} \rightarrow \mathbb{R}$ is $\Gamma$-periodic, i.e. assumed to satisfy

$$
V\left(x+b_{j}\right)=V(x), \quad j=1,2, \quad V \in \mathrm{L}^{\infty}(\Omega),
$$

and the magnetic potential $A: \mathbb{R}^{2} \rightarrow \mathbb{R}^{2}$ is also $\Gamma$-periodic and

$$
A \in \mathrm{C}_{\mathrm{per}}^{1}\left(\Omega ; \mathbb{R}^{2}\right), \quad \operatorname{div} A=0, \quad \int_{\Omega} A(x) d x=0 .
$$

Note that the last two conditions can be imposed without loss of generality; see Remark 2.3. The metric $g$ is a $\Gamma$-periodic symmetric $(2 \times 2)$-matrix function satisfying

$$
g \in \mathrm{C}_{\mathrm{per}}^{2}\left(\Omega ; \mathrm{M}_{2}(\mathbb{R})\right), \quad g(x) \geqslant m_{g} \mathbf{1}>0, \quad \text { where } \mathbf{1}=\left(\begin{array}{cc}
1 & 0 \\
0 & 1
\end{array}\right)
$$

for some positive constant $m_{g}$. The operator (2.1) is self-adjoint on $\mathrm{L}^{2}\left(\mathbb{R}^{2}\right)$ with the domain being the Sobolev space $H^{2}\left(\mathbb{R}^{2}\right)$. From the standard Floquet-Bloch theory (see, for example, [21, §XIII.16] or [13, §4.5]), it follows that $H$ is unitarily equivalent to the direct integral

$$
\int_{\tilde{\Omega}}^{\oplus} H(k) d k
$$


where $\widetilde{\Omega} \in \mathbb{R}^{2}$ is an elementary cell $\mathbb{R}^{d} / \Gamma^{\prime}$ of the dual lattice

$$
\Gamma^{\prime}=\left\{m_{1} b_{1}^{\prime}+m_{2} b_{2}^{\prime}: m_{1}, m_{2} \in 2 \pi \mathbb{Z}\right\}, \quad\left\langle b_{i}, b_{j}^{\prime}\right\rangle=\delta_{i j},
$$

and the $\left(m\right.$-sectorial) operators $H(k)$ in $\mathrm{L}^{2}(\Omega)$ are defined on the domain $H_{\text {per }}^{2}(\Omega)$ by

$$
H(k)=(-i \nabla+\bar{k}-A)^{*} g(-i \nabla+k-A)+V, \quad k \in \mathbb{C}^{2} .
$$

The family (2.7) is an analytic-type-A operator family with a compact resolvent, in the sense of [8]. This means that the domains Dom $H(k)$ do not depend on $k$, and $H(k) u$ is a (weakly) analytic vector-valued function of $k_{1}$ and $k_{2}$ for any $u \in \operatorname{Dom} H(k)=H_{\text {per }}^{2}(\Omega)$. Note that, while (2.5) and the statement of the main result only use real values of $k$, we will often need to consider (2.7) for $k \in \mathbb{C}^{2}$, and we need $\bar{k}$ in the definition to keep the expression analytic.

For $k \in \mathbb{R}^{2}$, let us denote the eigenvalues of $H(k)$, taken in the non-decreasing order, by $\lambda_{j}(k)$. These eigenvalues, considered as functions of $k$, are called band functions. These functions are $\Gamma^{\prime}$-periodic and piecewise real analytic on $\mathbb{R}^{2}$. The spectrum of $H$,

$$
\sigma(H)=\bigcup_{j}\left[\lambda_{j}^{-}, \lambda_{j}^{+}\right]
$$

is the union of the spectral bands $\left[\lambda_{j}^{-}, \lambda_{j}^{+}\right]$which are the ranges of $\lambda_{j}(\cdot)$. It is well known (under much wider assumptions than ours; see [2] and [22]) that there are no degenerate bands, i.e. we always have $\lambda_{j}^{-}<\lambda_{j}^{+}$. The bands, however, can overlap. Our main result concerns the structure of the extrema of band functions.

THEOREM 2.1. Let $H$ be the operator (2.1) with the potentials and the metric satisfying (2.2)-(2.4). Let $\lambda_{*}$ be a global minimal or maximal value of $\lambda_{j}(\cdot)$. Then, the level set

$$
\left\{k \in \widetilde{\Omega}: \lambda_{j}(k)=\lambda_{*}\right\}
$$

is finite.

The following Liouville theorem at the edge of the spectrum follows immediately from Theorem 2.1; see [17, Theorem 23 and Remark 6.1] or [18, Theorem 4.4].

Corollary 2.2. Let $H$ be the operator (2.1) with the potentials and the metric satisfying (2.2)-(2.4). Then, for every fixed $\lambda_{*} \in \partial(\sigma(H))$ and $n \in \mathbb{N}$, the space of solutions of

$$
(-i \nabla-A(x))^{*} g(x)(-i \nabla-A(x)) u(x)+V(x) u(x)=\lambda_{*} u(x)
$$

satisfying

$$
|u(x)|=O\left((1+|x|)^{n}\right)
$$

has finite dimension (which may depend on $\lambda_{*}$ and $n$ ). 
Remark 2.3. The second and third conditions from (2.3) can be imposed without loss of generality using a gauge transformation

$$
A \longmapsto A-\nabla \Phi-|\Omega|^{-1} \int_{\Omega} A(x) d x
$$

(see, for example, $[3, \S 1.2]$ ) with $\Phi$ periodic. The addition of $-\nabla \Phi$ is a unitary equivalence transformation of $H(k)$ for all $k$, and the addition of the last term is equivalent to the change of the quasi-momentum

$$
k \longmapsto k-|\Omega|^{-1} \int_{\Omega} A(x) d x
$$

Neither of these changes affects the main result.

The structure of the paper. In $\S \S 3-5$, we deal with the case of the scalar metric $g(x)=\omega^{2}(x) \mathbf{1}$. The proof is based on an identity from [7]. This identity shows that the values of $k_{1}$ such that $\lambda\left(k_{1} e_{1}+k_{2} e_{2}\right)=\lambda$ are eigenvalues of a certain non-self-adjoint operator $T_{1}\left(k_{2}, \lambda\right)$ (see Proposition 3.1 below). Our main observation is that the band edges correspond to degenerate eigenvalues of that operator. In $\S 3$, we introduce the operator $T_{1}$ and formulate the main technical result (Theorem 3.3), which shows that the set of the values of $k_{2}$ for which $T_{1}\left(k_{2}, \lambda\right)$ may have degenerate eigenvalues is discrete. Theorem 3.3 immediately implies the main result. In $\S 4$, we show that the condition for the operator $T_{1}\left(k_{2}, \lambda\right)$ to have degenerate eigenvalues is an analytic-type condition. Hence, either the set of "degenerate" $k_{2}$ is discrete, or the operator $T_{1}\left(k_{2}, \lambda\right)$ has degenerate eigenvalues for all $k_{2} \in \mathbb{C}$. In $\S 5$, we show that the latter case is impossible for the free operator and hence, using perturbation theory and estimates on the symbol, for the perturbed operator. $\S 6$ describes the reduction of the case of a general $\mathrm{C}^{2}$-metric to the case of a scalar one. In $\S 7$, we give an example of a discrete periodic Schrödinger operator for which the statement of the main theorem fails.

\section{The operator $T_{1}\left(k_{2}, \lambda\right)$}

In this section, we deal with the operator family

$$
H(k)=(-i \nabla+\bar{k}-A)^{*} \omega^{2}(-i \nabla+k-A)+V,
$$

which is a particular case of (2.7); here $\omega$ is a scalar function satisfying

$$
\omega \in \mathrm{C}_{\mathrm{per}}^{2}(\Omega) \text { and } \omega^{2} \geqslant m_{g}>0 .
$$


Let $\left\{e_{1}, e_{2}\right\}$ be a standard basis in $\mathbb{R}^{2}$. We also denote the coordinates of $k$ by $k_{1}$ and $k_{2}$, that is, $k=k_{1} e_{1}+k_{2} e_{2}$, and we will often denote $H(k)=H\left(k_{1} e_{1}+k_{2} e_{2}\right)$ by $H\left(k_{1}, k_{2}\right)$.

Since the statement of the main result is invariant under rotations and dilations of $\mathbb{R}^{2}$, we can fix the following choice of basis of the dual lattice:

$$
b_{1}^{\prime}=\alpha e_{1} \text { and } b_{2}^{\prime}=\beta e_{1}+e_{2}, \quad \text { where } \alpha, \beta \in \mathbb{R} .
$$

In the Hilbert space $H_{\text {per }}^{1}(\Omega) \oplus \mathrm{L}^{2}(\Omega)$, consider the following unbounded non-selfadjoint operator family:

$$
T_{1}\left(k_{2}, \lambda\right):=\left(\begin{array}{cc}
0 & \omega^{-2} I \\
-\left(H\left(0, k_{2}\right)-\lambda\right) & 2\left(i \partial_{1}+A_{1}\right)-2 i \omega^{-1} \partial_{1} \omega
\end{array}\right), \quad k_{2}, \lambda \in \mathbb{C},
$$

where $\operatorname{Dom}\left(T_{1}\left(k_{2}, \lambda\right)\right)=H_{\text {per }}^{2}(\Omega) \oplus H_{\mathrm{per}}^{1}(\Omega)$, and $\partial_{1}=\partial / \partial x_{1}$.

The operator $T_{1}\left(k_{2}, \lambda\right)$ is introduced in order to "linearize" the equation

$$
H\left(k_{1}, k_{2}\right) u=\lambda u,
$$

considered as a quadratic eigenvalue problem in $k_{1}$, similarly to [7, Lemma 3]. We summarize the properties of the family $T_{1}$ (most of which were also used in [7]) in the following proposition.

Proposition 3.1. The operators $T_{1}\left(k_{2}, \lambda\right)$ satisfy the following properties.

(i) For all $k_{2}, \lambda \in \mathbb{C}$, the operator $T_{1}\left(k_{2}, \lambda\right)$ is closed on the domain $H_{\mathrm{per}}^{2}(\Omega) \oplus H_{\mathrm{per}}^{1}(\Omega)$. As a consequence, the family $T_{1}(\cdot, \lambda)$ is an analytic-type- $A$ operator family.

(ii) Suppose that $\lambda \notin \sigma\left(H\left(k_{1}, k_{2}\right)\right)$. Then, $k_{1} \notin \sigma\left(T_{1}\left(k_{2}, \lambda\right)\right)$, and the resolvent

$$
\left(T_{1}\left(k_{2}, \lambda\right)-k_{1}\left(\begin{array}{ll}
I & 0 \\
0 & I
\end{array}\right)\right)^{-1}
$$

is compact in $H_{\mathrm{per}}^{1}(\Omega) \oplus \mathrm{L}^{2}(\Omega)$.

(iii) $k_{1} \in \sigma\left(T_{1}\left(k_{2}, \lambda\right)\right)$ if and only if $\lambda \in \sigma(H(k))$, where $k=k_{1} e_{1}+k_{2} e_{2}$.

(iv) For all $k_{2}, \lambda \in \mathbb{C}$, the set $\sigma\left(T_{1}\left(k_{2}, \lambda\right)\right)$ is discrete in $\mathbb{C}$ and $2 \pi \alpha$-periodic, where $\alpha$ is defined in (3.3).

Proof. Part (i). Clearly, we have that the operator $T_{1}\left(k_{2}, \lambda\right)$ is bounded as an operator from $H_{\text {per }}^{2}(\Omega) \oplus H_{\text {per }}^{1}(\Omega)$ to $H_{\text {per }}^{1}(\Omega) \oplus \mathrm{L}^{2}(\Omega)$. We also have

$$
\begin{aligned}
& \left\|T_{1}\left(k_{2}, \lambda\right)\left(\begin{array}{c}
u \\
v
\end{array}\right)\right\|_{H_{\mathrm{per}}^{1}(\Omega) \oplus \mathrm{L}^{2}(\Omega)}^{2} \\
& \quad=\left\|\omega^{-2} v\right\|_{H_{\mathrm{per}}^{1}(\Omega)}^{2}+\left\|-\left(H\left(0, k_{2}\right)-\lambda\right) u+\left(2\left(i \partial_{1}+A_{1}\right)-2 i \omega^{-1} \partial_{1} \omega\right) v\right\|_{L^{2}(\Omega)}^{2},
\end{aligned}
$$


from which it follows that the convergence in $T_{1}\left(k_{2}, \lambda\right)$-norm implies convergence of $v$ in $H^{1}$ and convergence of $u$ in $H^{2}$, so that $T_{1}\left(k_{2}, \lambda\right)$ is closed on its domain. Strong analyticity in $k_{2}$ and $\lambda$ follows directly from the definition.

Part (ii). Suppose that $\lambda \notin \sigma(H(k))$. Then, the equation

$$
\left(T_{1}\left(k_{2}, \lambda\right)-k_{1}\left(\begin{array}{ll}
I & 0 \\
0 & I
\end{array}\right)\right)\left(\begin{array}{l}
u \\
v
\end{array}\right)=\left(\begin{array}{l}
f \\
g
\end{array}\right)
$$

has a unique solution $\left(\begin{array}{l}u \\ v\end{array}\right)$ given by

$$
\begin{aligned}
& u=(H(k)-\lambda)^{-1}\left\{\left(2 i \partial_{1}+2 A_{1}-2 i \omega^{-1} \partial_{1} \omega-k_{1}\right) \omega^{2} f-g\right\}, \\
& v=\omega^{2}\left(f+k_{1} u\right) .
\end{aligned}
$$

Let $R(k, \lambda)=(H(k)-\lambda)^{-1}$. By plugging the expression for $u$ into the second equation of (3.6), we can rewrite (3.6) in the operator form, applied to a vector

$$
\left(\begin{array}{l}
f \\
g
\end{array}\right) \in H_{\mathrm{per}}^{1}(\Omega) \oplus \mathrm{L}^{2}(\Omega)
$$

as follows:

$$
\begin{aligned}
& \left(T_{1}\left(k_{2}, \lambda\right)-k_{1}\left(\begin{array}{ll}
I & 0 \\
0 & I
\end{array}\right)\right)^{-1}\left(\begin{array}{l}
f \\
g
\end{array}\right) \\
& =\left(\begin{array}{cc}
0 & 0 \\
\omega^{2} I & 0
\end{array}\right)\left(\begin{array}{l}
f \\
g
\end{array}\right)
\end{aligned}
$$

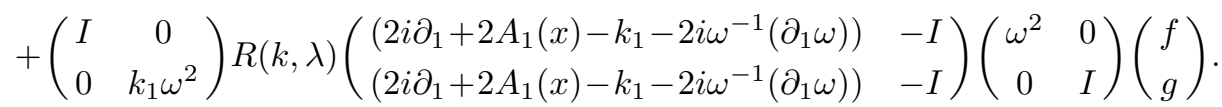

The first operator in the right-hand side is compact in $H_{\mathrm{per}}^{1}(\Omega) \oplus \mathrm{L}^{2}(\Omega)$, because the embedding $H_{\mathrm{per}}^{1}(\Omega) \subset \mathrm{L}^{2}(\Omega)$ is compact. The operator in the second term is compact, since $R(k, \lambda)$ is bounded as an operator from $\mathrm{L}^{2}(\Omega)$ to $H_{\mathrm{per}}^{2}(\Omega)$, and hence is compact from $\mathrm{L}^{2}(\Omega)$ to $H_{\mathrm{per}}^{1}(\Omega)$. Hence, the resolvent of $T_{1}\left(k_{2}, \lambda\right)$ is compact, which completes the proof of part (ii).

Part (iii). The "only if" part is included in Part (ii). To establish the "if" part, suppose that $H(k) u=\lambda u$. Then, $u \in H_{\mathrm{per}}^{2}(\Omega)$, and

$$
T_{1}\left(k_{2}, \lambda\right)\left(\begin{array}{c}
u \\
k_{1} \omega^{2} u
\end{array}\right)=k_{1}\left(\begin{array}{c}
u \\
k_{1} \omega^{2} u
\end{array}\right) .
$$


This completes the proof of (iii).

Part (iv). From the proofs of the absolute continuity of the spectrum (see, e.g., [2]), it follows that, for any $\lambda, k_{2} \in \mathbb{C}$, the set $\left\{k_{1}: \lambda \in \sigma\left(H\left(k_{1}, k_{2}\right)\right)\right\}$ is discrete. Hence, there exists at least one value of $k_{1}$ such that the resolvent (3.5) exists, which, together with Part (ii), implies that $\sigma\left(T\left(k_{2}, \lambda\right)\right)$ is discrete. Periodicity of the spectrum follows from the fact that $H(k)$ is unitarily equivalent to $H\left(k+b^{\prime}\right)$ for any $b^{\prime} \in \Gamma^{\prime}$ (see (2.6)), and so $H\left(k_{1}, k_{2}\right)$ is unitarily equivalent to $H\left(k_{1}+2 \pi \alpha, k_{2}\right)$.

In the sequel, by "the multiplicity of an isolated eigenvalue" we will mean algebraic multiplicity, i.e. the dimension of the range of the corresponding Riesz projection. We will call an eigenvalue degenerate if its algebraic multiplicity is greater than or equal to 2 . Otherwise, an eigenvalue is called simple.

LEMma 3.2. Suppose that a band function $\lambda_{j}(\cdot)$ attains its local minimum or maximum value $\lambda_{*}$ at $k^{*}=k_{1}^{*} e_{1}+k_{2}^{*} e_{2} \in \mathbb{R}^{2}$. Then, $k_{1}^{*}$ is an eigenvalue of $T_{1}\left(k_{2}^{*}, \lambda_{*}\right)$ with (algebraic) multiplicity at least 2 .

Proof. By Proposition 3.1, $k_{1}^{*}$ is an eigenvalue of $T_{1}\left(k_{2}^{*}, \lambda_{*}\right)$. For some $\varepsilon>0$, there are no other eigenvalues of $T_{1}\left(k_{2}^{*}, \lambda_{*}\right)$ within the closed disc $\overline{B_{\varepsilon}\left(k_{1}^{*}\right)}$. Let

$$
P\left(k_{2}^{*}, \lambda\right):=-\frac{1}{2 \pi i} \oint_{\partial B_{\varepsilon}\left(k_{1}^{*}\right)}\left(T_{1}\left(k_{2}, \lambda\right)-\varkappa I\right)^{-1} d \varkappa
$$

be the Riesz projection. The standard arguments [8, $\S$ IV.3.5] show that, for some $\delta>0$, $\operatorname{rank} P\left(k_{2}^{*}, \lambda\right)$ is continuous in $\lambda$ as long as $\left|\lambda-\lambda_{*}\right|<\delta$. Without loss of generality, assume that $k^{*}$ is a local minimum of $\lambda_{j}(\cdot)$.

From the proofs of the absolute continuity of the spectrum (see, e.g., [2]), it follows that $\lambda_{j}(k)$ cannot be constant in $k_{1}$ on any interval. Then, for a sufficiently small $\delta>0$, the equation $\lambda_{j}\left(k_{1}, k_{2}^{*}\right)=\lambda_{*}+\delta$ has at least two different solutions as an equation in $k_{1}$ (note that these arguments do not use any analyticity of $\lambda_{j}\left(\cdot, k_{2}^{*}\right)$, only continuity). Hence, by part (iii) of Proposition $3.1, \operatorname{rank} P\left(k_{2}^{*}, \lambda_{*}+\delta\right) \geqslant 2$ for all sufficiently small $\delta$, and therefore $\operatorname{rank} P\left(k_{2}^{*}, \lambda_{*}\right) \geqslant 2$, due to continuity.

The following is the main technical result of the paper.

TheOREM 3.3. Suppose that the coefficients $\omega, A$ and $V$ satisfy (3.2), (2.3) and (2.2). For any $\lambda \in \mathbb{R}$, the set

$\left\{k_{2} \in \mathbb{R}:\right.$ the operator $T_{1}\left(k_{2}, \lambda\right)$ has at least one real degenerate eigenvalue $\}$ is discrete. 
Proof of Theorem 2.1: the case of a scalar metric. Fix a band function $\lambda_{j}(\cdot)$ and assume that $\lambda_{*}$ is a minimum or a maximum of $\lambda_{j}$. From Theorem 3.3 and Lemma 3.2, the set of possible $k_{2}$ such that for some $k_{1}$ we have $\lambda_{j}(k)=\lambda_{*}$ is discrete. For each of these $k_{2}$, the set of possible values of $k_{1}$ is also discrete by Proposition 3.1.

Theorem 3.3 is proved in $\S 4$ and $\S 5$. The rest of the proof of Theorem 2.1 is a (mostly standard) argument of transforming a general metric to a scalar metric by introducing isothermal coordinates. This is done in $\S 6$.

\section{Proof of Theorem 3.3}

Let

$$
p(z)=z^{n}+a_{n-1} z^{n-1}+\ldots+a_{0}
$$

be a monic polynomial with roots $z_{1}, \ldots, z_{n}$. The discriminant of $p$ is defined as

$$
\Delta(p)=\prod_{1 \leqslant i<j \leqslant n}\left(z_{i}-z_{j}\right)^{2}
$$

It is clear that $\Delta(p)$ vanishes if and only if $p$ has roots of multiplicity greater than or equal to 2. It is well known (see, for example, $[26, \S 5.9]$ ) that $\Delta(p)$ is a polynomial function of the coefficients $a_{0}, \ldots, a_{n-1}$.

The proof of the following lemma can be extracted from a slightly different and more abstract setting of [13] and [27]. For the convenience of the reader, we include the argument.

Lemma 4.1. Suppose that $\mathcal{C}$ is a simple closed piecewise smooth contour in $\mathbb{C}$, and let $\{T(z): z \in \mathcal{D}\}$ be an operator family of type $A$ in a Hilbert space $\mathcal{H}$ analytic in a simply connected domain $\mathcal{D} \subset \mathbb{C}$. Suppose that, for all $z \in \mathcal{D}$, the spectrum of $T(z)$ in the interior of $\mathcal{C}$ is discrete and finite, and $\sigma(T(z)) \cap \mathcal{C}=\varnothing$. Then, the set

$$
\{z \in \mathcal{D}: T(z) \text { has at least one degenerate eigenvalue in the interior of } \mathcal{C}\}
$$

is a null-set of a function analytic in $\mathcal{D}$, and hence this set either coincides with $\mathcal{D}$ or is discrete in $\mathcal{D}$.

Proof. Let

$$
P(z):=-\frac{1}{2 \pi i} \oint_{\mathcal{C}}(T(z)-\varkappa I)^{-1} d \varkappa
$$

be the Riesz projection. By assumption, $n:=\operatorname{rank} P(z)=$ const is finite and independent on $z$, and $P(z)$ is analytic in $\mathcal{D}$. Fix $z_{0} \in \mathcal{D}$. The results of $[8, \S$ VII.1.3] imply that 
there exists a bounded operator-valued function $U: \mathcal{D} \rightarrow \mathcal{B}(\mathcal{H})\left({ }^{1}\right)$ analytic in $\mathcal{D}$, such that $U(\cdot)^{-1}$ is also analytic in $\mathcal{D}$ and $P(z)=U(z) P\left(z_{0}\right) U(z)^{-1}$. Take

$$
T_{0}(z):=\left.U(z)^{-1} T(z) U(z)\right|_{\operatorname{ran} P\left(z_{0}\right)}
$$

The family $T_{0}(z)$ is an analytic operator family acting in a fixed finite-dimensional space that has the same eigenvalues and multiplicities as $T(z)$ restricted to $\operatorname{ran} P(z)$. The monic polynomial $p_{z}(\varkappa)=(-1)^{n} \operatorname{det}\left(T_{0}(z)-\varkappa\right)$ is the characteristic polynomial of $T_{0}(z)$ and has the coefficients analytic in $\mathcal{D}$ (in the variable $z$ ). Hence, its discriminant $\Delta\left(p_{z}\right)$ is also an analytic function in $\mathcal{D}$ vanishing if and only if $T_{0}(z)$ (and, as a consequence, $T(z))$ has degenerate eigenvalues in the interior of $\mathcal{C}$.

Recall that we had a special choice of basis in $\Gamma^{\prime}$,

$$
b_{1}^{\prime}=\alpha e_{1} \quad \text { and } \quad b_{2}^{\prime}=\beta e_{1}+e_{2} .
$$

Let also

$$
k=k_{1} e_{1}+k_{2} e_{2}, \quad k_{1}=r_{1}+i l_{1} \quad \text { and } \quad k_{2}=r_{2}+i l_{2} .
$$

The following two theorems are the main technical statements of the paper. We postpone the proofs to the next section.

Without loss of generality, one may assume that $\lambda=0$, by possibly choosing a different $V$. In the sequel, we will make this assumption and drop $\lambda$ from the notation for $T_{1}$, that is, $T_{1}\left(k_{2}\right):=T_{1}\left(k_{2}, 0\right)$.

Theorem 4.2. Let $\delta>0$. There exist $C=C(A, V, \omega)$ and $C_{1}=C_{1}(A, V, \omega, \delta) \in 2 \pi \mathbb{Z}$ such that operator $H(k)$ defined in (3.1) is invertible and satisfies

$$
\left\|H(k)^{-1}\right\| \leqslant \frac{C}{\left|l_{1}\right| \delta^{2}},
$$

provided that $\operatorname{dist}\left(r_{2}, 2 \pi \mathbb{Z}\right) \geqslant \delta, l_{1} \in 2 \pi \mathbb{Z},\left|l_{1}\right| \geqslant C_{1}$. As a consequence, the horizontal lines $\operatorname{Im} k_{1}= \pm C_{1}$ have empty intersection with $\sigma\left(T_{1}\left(k_{2}\right)\right)$.

TheOREM 4.3. There exists $l=l(A, V, \omega) \in 2 \pi \mathbb{Z}$ such that, for all $n \in 2 \pi \mathbb{Z}$, the spectrum of $T_{1}\left(k_{2}\right)$ is simple for $k_{2}=\frac{1}{2} \pi+n+i\left(\frac{1}{2} \pi+l\right) \alpha$.

Proof of Theorem 3.3. Assume the contrary, i.e. that the set of $k_{2} \in \mathbb{R}$ for which $T_{1}\left(k_{2}\right)$ has real degenerate eigenvalues has a limit point $k_{2}^{(0)}$ (as above, we assume $\lambda=0$ ). Let us consider two cases.

$\left({ }^{1}\right) \mathcal{B}(\mathcal{H})$ denotes the algebra of bounded operators on a Hilbert space $H$. 
Case 1. Suppose that $\operatorname{dist}\left(k_{2}^{(0)}, 2 \pi \mathbb{Z}\right)>0$. Take $\delta=\min \left\{\frac{1}{2} \pi, \operatorname{dist}\left(k_{2}^{(0)}, 2 \pi \mathbb{Z}\right)\right\}$. There exists a single $n \in 2 \pi \mathbb{Z}$ such that $k_{2}^{(0)} \in[n+\delta, n+2 \pi-\delta]$. Let $\mathcal{C}_{0}$ be a path in the $k_{2}$-plane starting at $k_{2}^{(0)}$, then going straight towards the point $\frac{1}{2} \pi+n$, and then going vertically towards the point $k_{2}^{(1)}:=\frac{1}{2} \pi+n+i\left(\frac{1}{2} \pi+l\right) \alpha$ from Theorem 4.3.

The points $k_{2} \in \mathcal{C}_{0}$ satisfy the assumptions of Theorem 4.2. Let us consider the eigenvalues of $T_{1}\left(k_{2}\right)$ lying within the strip $\left|\operatorname{Im} k_{1}\right|<C_{1}$, where $C_{1}$ is the constant from Theorem 4.2. They form a discrete $2 \pi \alpha$-periodic set. For each $k_{2} \in \mathcal{C}_{0}$, there exists a point $r\left(k_{2}\right) \in \mathbb{R}$ which is not a real part of any of these eigenvalues. Moreover, by continuity arguments, this also holds in a small (complex) neighbourhood of $k_{2}$. Let us cover $\mathcal{C}_{0}$ by a finite number of these neighbourhoods $\mathcal{D}_{j}, j=1, \ldots, p$, so that $k_{2}^{(0)} \in \mathcal{D}_{1}$ and $k_{2}^{(1)} \in \mathcal{D}_{p}$, and denote the corresponding values of $r\left(k_{2}\right)$ by $r_{j}$. For each $j$, denote by $\mathcal{C}_{j}$ the boundary of the following rectangle:

$$
r_{j}<\operatorname{Re} k_{1}<r_{j}+2 \pi \alpha \quad \text { and } \quad-C_{1}<\operatorname{Im} k_{1}<C_{1} \text {. }
$$

Informally speaking, each rectangle contains all eigenvalues that we are interested in: they are initially on the real line, they cannot cross the lines $\operatorname{Im} k_{1}= \pm C_{1}$, and the pictures to the right and to the left copy the picture in the rectangle, due to periodicity.

Let us apply Lemma 4.1 to each of the domains $\mathcal{D}_{j}$ and contours $\mathcal{C}_{j}$. Due to Theorem 4.3 , the spectrum of $T_{1}\left(k_{2}^{(1)}\right)$ is simple, and hence the set of "degenerate" $k_{2}$ should be discrete in a neighbourhood $\mathcal{D}_{p}$ of $k_{2}^{(1)}$. By the standard arguments of analytic continuation, it should also be discrete in every neighbourhood $\mathcal{D}_{1}, \ldots, \mathcal{D}_{p}$. However, since $k_{2}^{(0)} \in \mathcal{D}_{1}$, it is not discrete in $\mathcal{D}_{1}$, which is a contradiction.

Case 2. Suppose that $k_{2}^{(0)} \in 2 \pi \mathbb{Z}$. The set of real eigenvalues of $T_{1}\left(k_{2}^{(0)}\right)$ is, again, discrete and $2 \pi \alpha$-periodic. Let us surround the eigenvalues on one period by a contour $\mathcal{C}$ containing no other eigenvalues. In a small neighbourhood $\mathcal{D}_{0}$ of $k_{2}^{(0)}$, these eigenvalues still stay within $\mathcal{C}$. Apply Lemma 4.1 to $\mathcal{C}$ and $\mathcal{D}_{0}$. Again, since the set of "degenerate" values of $k_{2}$ is not discrete in $\mathcal{D}_{0}$, it should coincide with $\mathcal{D}_{0}$, and hence there exists at least one more point with the same property that belongs to $\mathbb{R} \backslash 2 \pi \mathbb{Z}$, and thus the situation reduces to Case 1 .

\section{Proofs of Theorems 4.2 and 4.3}

Let us start by recalling some notation introduced above:

$$
\begin{gathered}
b_{1}^{\prime}=\alpha e_{1} \text { and } b_{2}^{\prime}=\beta e_{1}+e_{2}, \quad \alpha, \beta \in \mathbb{R} ; \\
k=k_{1} e_{1}+k_{2} e_{2}, \quad k_{1}=r_{1}+i l_{1} \text { and } k_{2}=r_{2}+i l_{2}, \quad r_{1}, r_{2}, l_{1}, l_{2} \in \mathbb{R} .
\end{gathered}
$$


In this section, we will emphasize the dependence of $H$ on $g, A$ and $V$, and use the notation $H(k ; g, A, V)$. Consider the free operator $H_{0}(k):=H(k ; \mathbf{1}, 0,0)$. Its eigenfunctions are of the form

$$
\begin{gathered}
\exp \{i m \cdot x\}=\exp \left\{i\left(m_{1} b_{1}^{\prime}+m_{2} b_{2}^{\prime}\right) \cdot\left(x_{1} e_{1}+x_{2} e_{2}\right)\right\}=\exp \left\{i\left(\left(\alpha m_{1}+\beta m_{2}\right) x_{1}+m_{2} x_{2}\right)\right\}, \\
m=m_{1} b_{1}^{\prime}+m_{2} b_{2}^{\prime} \in \Gamma^{\prime}, \quad m_{1}, m_{2} \in 2 \pi \mathbb{Z},
\end{gathered}
$$

and

$$
H_{0}(k) \exp \{i m \cdot x\}=\left(\left(-i \partial_{1}+k_{1}\right)^{2}+\left(-i \partial_{2}+k_{2}\right)^{2}\right) \exp \{i m \cdot x\}=h_{m}(k) \exp \{i m \cdot x\},
$$

where $h_{m}(k)$ is the symbol of $H_{0}(k)$ :

$$
\begin{aligned}
h_{m}(k) & =\left(\alpha m_{1}+\beta m_{2}+k_{1}\right)^{2}+\left(m_{2}+k_{2}\right)^{2}=q_{m}^{+}(k) q_{m}^{-}(k), \\
q_{m}^{ \pm}(k) & =\alpha m_{1}+\beta m_{2}+r_{1} \mp l_{2}+i\left(l_{1} \pm m_{2} \pm r_{2}\right) .
\end{aligned}
$$

Let also $Q^{ \pm}(k)$ be the operators with symbols $q_{m}^{ \pm}(k)$, respectively, so that

$$
H_{0}(k)=Q^{+}(k) Q^{-}(k) \text {. }
$$

Suppose that the magnetic potential $A$ satisfies (2.3). Then, there exists a $\Gamma$-periodic scalar function $\varphi \in C_{\mathrm{per}}^{2}(\Omega)$ such that

$$
(\nabla \varphi)(x)=A_{2}(x) e_{1}-A_{1}(x) e_{2}, \quad \int_{\Omega} \varphi(x) d x=0, \quad\|\varphi\|_{\mathrm{C}^{2}(\Omega)} \leqslant C\|A\|_{\mathrm{C}^{1}(\Omega)} .
$$

Let also

$$
B(x)=\partial_{1} A_{2}(x)-\partial_{2} A_{1}(x), \quad w(x):=e^{-2 \varphi(x)} .
$$

The operator $H(k ; \mathbf{1}, A, B)$ is called the Pauli operator (more precisely, a block of the Pauli operator). The following is proved in [2] and allows us to reduce the case of the magnetic potential, essentially, to the case of the free operator.

Proposition 5.1. Under the above assumptions, if $Q^{+}(k)$ and $Q^{-}(k)$ are invertible, then $H(k ; \mathbf{1}, A, B)$ is also invertible, and

$$
\begin{aligned}
H(k ; \mathbf{1}, A, B)^{-1} & =e^{\varphi} Q^{-}(k)^{-1} e^{-2 \varphi} Q^{+}(k)^{-1} e^{\varphi} \\
& =e^{\varphi(x)} H_{0}(k)^{-1}\left(e^{-\varphi}+\left(-i \partial_{1} w+\partial_{2} w\right) Q^{+}(k)^{-1} e^{\varphi}\right) .
\end{aligned}
$$

The following proposition can also be easily verified; see [4]. It will be used to reduce the case of a scalar metric $g=\omega^{2} \mathbf{1}$ to the case $g=\mathbf{1}$. 
Proposition 5.2. Let $\omega \in \mathrm{C}_{\mathrm{per}}^{2}(\Omega), V \in \mathrm{L}^{\infty}(\Omega)$ and $A \in \mathrm{C}_{\mathrm{per}}^{1}(\Omega)$. Then,

$$
\begin{aligned}
& H\left(k ; \omega^{2} \mathbf{1}, A, V\right)=\omega H\left(k ; \mathbf{1}, A, \omega^{-2} V+\omega^{-1} \Delta \omega\right) \omega, \\
& \omega H(k ; \mathbf{1}, A, V) \omega=H\left(k ; \omega^{2} \mathbf{1}, A, \omega^{2} V-\omega \Delta \omega\right) .
\end{aligned}
$$

Proof of Theorem 4.2. Suppose that $\operatorname{dist}\left(r_{2}, 2 \pi \mathbb{Z}\right)=\delta$. Since $l_{1} \pm m_{2} \in 2 \pi \mathbb{Z}$, we have $\left|q_{m}^{ \pm}(k)\right| \geqslant \delta$. In addition, $\operatorname{Im} q_{m}^{+}(k)+\operatorname{Im} q_{m}^{-}(k)=2 l_{1}$, and hence we either have $\left|q_{m}^{+}(k)\right| \geqslant\left|l_{1}\right|$ or $\left|q_{m}^{-}(k)\right| \geqslant\left|l_{1}\right|$. Combining these estimates, we obtain $\left|h_{m}(k)\right| \geqslant\left|l_{1}\right| \delta$, and

$$
\left\|H_{0}(k)^{-1}\right\| \leqslant \frac{1}{\left|l_{1}\right| \delta} \quad \text { and } \quad\left\|Q^{+}(k)^{-1}\right\| \leqslant \frac{1}{\delta},
$$

which completes the proof for $A=0, V=0$ and $\omega=1$. If $A \neq 0$ and $V(x)=B(x)$, then, from (5.2) and (5.5), we get

$$
\left\|H(k ; \mathbf{1}, A, B)^{-1}\right\| \leqslant \frac{C}{\left|l_{1}\right| \delta^{2}},
$$

where $C$ depends on $A$ via $w$ and $\varphi$. The standard Neumann series arguments imply that the bound (5.6) holds for the operator $H(k, \mathbf{1}, A, V)$ with arbitrary $V \in \mathrm{L}^{\infty}(\Omega)$ (and maybe a different $C$ ) for sufficiently large $l_{1}$, say

$$
\left|l_{1}\right| \geqslant \frac{2\|V-B\|_{\mathrm{L}^{\infty}(\Omega)} C}{\delta^{2}} .
$$

The case of arbitrary $\omega$ follows from Proposition 5.2.

We now make some preparations for the proof of Theorem 4.3. Fix $k_{2}$ as in the formulation of the theorem, so that

$$
r_{2}=\frac{1}{2} \pi+n \text { and } l_{2}=\left(\frac{1}{2} \pi+l\right) \alpha, \quad l, n \in 2 \pi \mathbb{Z}
$$

For these $k_{2}$, define

$$
\Sigma_{n}:=\left\{k_{1} \in \mathbb{C}: h_{m}\left(k_{1}, k_{2}\right)=0 \text { for some } m_{1}, m_{2} \in 2 \pi \mathbb{Z}\right\}
$$

In other words, it is the set of $k_{1}$ for which $H_{0}\left(k_{1}, k_{2}\right)$ is not invertible. A simple computation shows that $\Sigma_{n}$ consists of points $r_{1}+i l_{1}$ of the following form:

$$
\left\{\begin{array}{l}
r_{1}=-\alpha m_{1}-\beta m_{2} \mp\left(\frac{1}{2} \pi+l\right) \alpha, \quad m_{1}, m_{2} \in 2 \pi \mathbb{Z} \\
l_{1}= \pm\left(\frac{1}{2} \pi+n+m_{2}\right)
\end{array}\right.
$$

Since one can replace the variables $m_{1}$ by $m_{1}+l$, one can see that the set $\Sigma_{n}$ does not depend on $l$. 


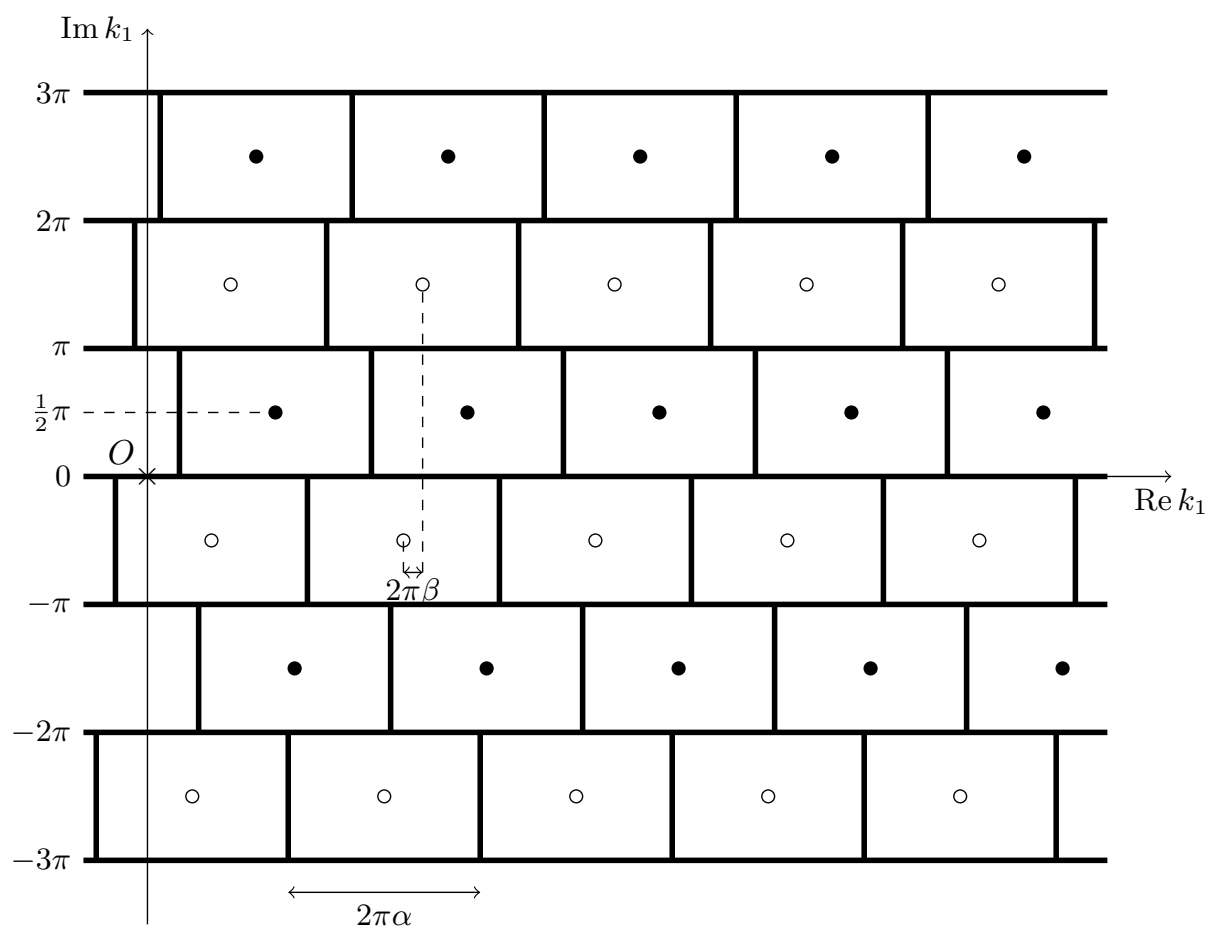

Figure 1. The sets $\Sigma_{n}$ and $G_{n}$.

Let us describe the set $\Sigma_{n}$ in more detail. First of all, it is easy to see that different values of $\left(m_{1}, m_{2}\right)$ give different points of $\Sigma_{n}$, as $m_{2}$ and the signs are uniquely determined by the value of $l_{1}$, and $m_{1}$ is determined by $r_{1}$ afterwards. Next, the set $\Sigma_{n}$ lies on the union of horizontal lines $\operatorname{Im} k_{1} \in \frac{1}{2} \pi+\pi \mathbb{Z}$. On each line, it is a sequence of equally spaced points with the spacings $2 \pi \alpha$.

We will also need another set $G_{n}$ defined by

$$
G_{n}:=(\mathbb{R}+i \pi \mathbb{Z}) \cup \bigcup_{z \in \Sigma_{n}}\left(z+\pi \alpha+i\left[-\frac{\pi}{2}, \frac{\pi}{2}\right]\right) .
$$

The set $G_{n}$ consists of horizontal lines $\operatorname{Im} k_{1} \in \pi \mathbb{Z}$ separating the horizontal lines of $\Sigma_{n}$. In addition, for each point of $\Sigma_{n}$, we include a vertical line segment of the length $\pi$ separating this point from the next point of $\Sigma_{n}$ lying on the same line. One can imagine $G_{n}$ as a "brick wall" consisting of rectangles such that there is exactly one element of $\Sigma_{n}$ inside of each rectangle.

In Figure 1, an example of $\Sigma_{n}$ and $G_{n}$ is shown for $n=0, \alpha=0.75$ and $\beta=0.075$. The set $G_{n}$ is represented by thick lines, and the locations of points of $\Sigma_{n}$ are indicated by black and white circles, corresponding to the upper or lower choice of signs in (5.8), respectively. 
LEMma 5.3. Suppose that $k_{1} \in G_{n}$ and $k_{2}=\frac{1}{2} \pi+n+i\left(\frac{1}{2} \pi+l\right) \alpha$, where $l, n \in 2 \pi \mathbb{Z}$. Then,

$$
\left|h_{m}(k)\right| \geqslant C|l|
$$

uniformly in $m_{1}, m_{2} \in 2 \pi \mathbb{Z}$.

Proof. Since $\left|\operatorname{Re} q_{m}^{+}(k)-\operatorname{Re} q_{m}^{-}(k)\right|=2\left|l_{2}\right| \geqslant C|l|$, we have for each $m$ either

$$
\left|q_{m}^{+}(k)\right| \geqslant \frac{1}{2} C|l| \quad \text { or } \quad\left|q_{m}^{-}(k)\right| \geqslant \frac{1}{2} C|l| .
$$

On the other hand, $\left|q_{m}^{+}(k)\right|$, as well as $\left|q_{m}^{-}(k)\right|$, is a distance between $k_{1}$ and some point on $\Sigma_{n}$, which implies the following lower bound:

$$
\left|q_{m}^{ \pm}(k)\right| \geqslant \operatorname{dist}\left(k_{1}, \Sigma_{n}\right) \geqslant \operatorname{dist}\left(G_{n}, \Sigma_{n}\right)=\min \left\{\frac{1}{2} \pi, \pi \alpha\right\}
$$

The combination of these estimates completes the proof of the lemma.

Remark 5.4. Lemma 5.3 is the main ingredient of the proof that relies on the assumption $d=2$. In $d \geqslant 3$, one cannot construct a set $G_{n}$ with similar properties and constant size of the bricks.

Corollary 5.5. Under the assumptions of Lemma 5.3, there exists $L_{0}(A, V, \omega)>0$ such that, if $|l|>L_{0}(\omega, A, V)$, then the operator $H\left(k ; \omega^{2} \mathbf{1}, A, V\right)$ is invertible and

$$
\left\|H\left(k ; \omega^{2} \mathbf{1}, A, V\right)^{-1}\right\| \leqslant \frac{C(\omega, A, V)}{|l|},
$$

where the constants $C$ and $L_{0}$ depend only on $\|A\|_{\mathrm{C}^{1}(\Omega)},\|V\|_{\mathrm{L}^{\infty}(\Omega)},\|\omega\|_{\mathrm{C}^{2}(\Omega)}$ and on the constant $m_{g}$ from (3.2).

Proof. From Proposition 5.2, we have

$$
\left\|H\left(k ; \omega^{2} \mathbf{1}, A, V\right)^{-1}\right\| \leqslant m_{g}^{-2}\left\|H\left(k ; \mathbf{1}, A, V_{\omega}\right)^{-1}\right\|,
$$

where

$$
V_{\omega}=\omega^{-2} V+\omega^{-1} \Delta \omega,
$$

and therefore

$$
\left\|V_{\omega}\right\|_{L^{\infty}(\Omega)} \leqslant m_{g}^{-2}\|V\|_{L^{\infty}(\Omega)}+m_{g}^{-1}\|\omega\|_{C^{2}(\Omega)} .
$$

From (5.2), (5.1), Lemma 5.3 and (5.9), we have

$$
\left\|H(k ; \mathbf{1}, A, B)^{-1}\right\| \leqslant C(A)\left\|H_{0}(k)^{-1}\right\| \leqslant \frac{C_{1}(A)}{|l|},
$$

where $C(A)$ and $C_{1}(A)$ depend only on $\|A\|_{\mathrm{C}^{1}(\Omega)}$. Since $\|B\|_{\mathrm{L}^{\infty}(\Omega)} \leqslant 2\|A\|_{\mathrm{C}^{1}(\Omega)}$, we can use the same Neumann series argument as in the proof of Theorem 4.2 to replace $B$ by $V_{\omega}$. 
Proof of Theorem 4.3. Denote by $T_{\mu}\left(k_{2}\right)$ the operator $T_{1}\left(k_{2}\right)$, with $V, A$ and $\omega$ replaced by $\mu V, \mu A$ and $\mu \omega+(1-\mu)$, respectively. It is a 1 -parameter family connecting the "free" operator $T_{0}\left(k_{2}\right)$ with $T_{1}\left(k_{2}\right)$.

It is easy to see that $\sigma\left(T_{0}\left(k_{2}\right)\right)=\Sigma_{n}$, because $\Sigma_{n}$ is exactly the set of $k_{1} \in \mathbb{C}$ for which the symbol of $H_{0}(k)$ is not invertible. Moreover, an easy computation shows that, for each $k_{1} \in \Sigma_{n}$, the corresponding eigenspace is 1-dimensional and is spanned by $\left(\begin{array}{c}e^{i m \cdot x} \\ k_{1} e^{i m \cdot x}\end{array}\right)$, where $m$ is determined by $k_{1}$ via (5.8). Note that each value of $m$ appears twice (for two different values of $k_{1}$ ) because of two possible signs. Hence, the total collection of eigenvectors spans $H_{\text {per }}^{1}(\Omega) \oplus \mathrm{L}^{2}(\Omega)$, so there are no Jordan cells and the spectrum of $T_{0}\left(k_{2}\right)$ is simple.

It remains to prove that $T_{1}\left(k_{2}\right)$ also has simple spectrum. Consider the Riesz projection of $T_{\mu}\left(k_{2}\right)$ with respect to the boundary of some rectangle of $G_{n}$. For $\mu=0$, the rectangle contains exactly one simple eigenvalue, and the range of the projection has dimension 1 . Let us increase $\mu$. The only way for the dimension of the range to change is to have an eigenvalue of $T_{\mu}\left(k_{2}\right)$ approach the set $G_{n}$. This, however, is impossible for $\mu \in[0,1]$, due to Corollary 5.5, and hence the eigenvalues of $T_{1}\left(k_{2}\right)$ is simple.

Remark 5.6. The proof of Theorem 4.3 is based on the ideas of $[7, \S \mathrm{VI}]$.

\section{The case of variable metric}

In this section we show how to reduce the case of an operator with arbitrary metric $g$ satisfying (2.4) to the case of the scalar metric. The technical difference with standard arguments such as in [22] is that we need to keep track of the quasi-momentum, in order to ensure that it is transformed linearly. This is done by an additional "gauge transformation". The following proposition establishes the existence of global isometric coordinates in which the metric $g$ becomes scalar. See [16, Proposition 18] for the proof.

Proposition 6.1. Suppose that $g$ satisfies (2.4). Then, there exists a basis $\left\{b_{1}^{*}, b_{2}^{*}\right\}$ of $\mathbb{R}^{2}$ and a one-to-one map $\Psi: \mathbb{R}^{2} \rightarrow \mathbb{R}^{2}, \Psi \in \mathrm{C}^{3}\left(\mathbb{R}^{2}\right)$, $\operatorname{det} \Psi^{\prime}(x) \neq 0$,

$$
\Psi(0)=0 \quad \text { and } \quad \Psi\left(x+n_{1} b_{1}+n_{2} b_{2}\right)=\Psi(x)+n_{1} b_{1}^{*}+n_{2} b_{2}^{*} \text { for all } n_{1}, n_{2} \in \mathbb{Z},
$$

such that

$$
\left|\operatorname{det} \Psi^{\prime}(x)\right|^{-1} \Psi^{\prime}(x) g(x) \Psi^{\prime}(x)^{t}=\omega^{2}(\Psi(x)) \mathbf{1},
$$

where $\omega \in C^{2}\left(\mathbb{R}^{2}\right)$ is a strictly positive scalar function periodic with respect to the lattice $\Gamma_{*}$ spanned by $b_{1}^{*}$ and $b_{2}^{*}$. 
Let us introduce some notation. Suppose that the operator $H(g, A, V)$ satisfies the assumptions of Theorem 2.1. Let $\Psi$ be the transformation obtained from Proposition 6.1. Denote by $T_{*}: \mathbb{R}^{2} \rightarrow \mathbb{R}^{2}$ the linear transformation defined by $T_{*}\left(b_{1}\right)=b_{1}^{*}$ and $T_{*}\left(b_{2}\right)=b_{2}^{*}$. The transformation $T_{*}$, as well as the map $\Psi$, transforms the lattice $\Gamma$ into $\Gamma_{*}$. Let also

$$
y=\Psi(x), \quad A_{*}(y)=\left(\Psi^{\prime}(x)^{-1}\right)^{t} A(x), \quad V_{*}(y)=\psi_{*}(y)^{-2} V(x), \quad \psi_{*}(y)=\left|\operatorname{det} \Psi^{\prime}(x)\right|^{1 / 2} .
$$

Let also

$$
\Omega_{\Psi}=\Psi(\Omega) \quad \text { and } \quad \Omega_{*}=\left\{y_{1} b_{1}^{*}+y_{2} b_{2}^{*}: y_{1}, y_{2} \in[0,1)\right\} .
$$

Note that both $\Omega_{*}$ and $\Omega_{\Psi}$ are fundamental domains of $\Gamma_{*}$, and there is a natural correspondence between $L^{2}\left(\Omega_{*}\right)$ and $L^{2}\left(\Omega_{\Psi}\right)$, as both can be identified with $\mathbb{R}^{d} / \Gamma_{*}$.

LEMMA 6.2. In the above notation, let $\Phi: \mathrm{L}^{2}(\Omega) \rightarrow \mathrm{L}^{2}\left(\Omega_{*}\right)$ be the unitary operator of change of variables

$$
u(x)=\psi_{*}(y)(\Phi u)(y), \quad y=\Psi(x),
$$

where $u$ is considered as an element of $L^{2}\left(\Omega_{*}\right)$. Then,

$$
\Phi H(0 ; g, A, V) \Phi^{-1}=\psi_{*} H\left(0 ; \omega^{2} \mathbf{1}, A_{*}, V_{*}\right) \psi_{*} .
$$

Proof. Let $v=\Phi u$, and let us extend it $\Gamma_{*}$-periodically into $\mathbb{R}^{d}$. Then, due to (6.1) and the change of variable rule, the quadratic form of the left-hand side applied to $v$ is equal to

$$
\begin{aligned}
\left(H(0 ; g, A, V) \Phi^{-1} v, \Phi^{-1} v\right)_{\mathrm{L}^{2}(\Omega)} & (H(0 ; g, A, V) u, u)_{\mathrm{L}^{2}(\Omega)} \\
= & \int_{\Omega}\left\langle g(x)\left(-i \nabla_{x}-A(x)\right) u(x),\left(-i \nabla_{x}-A(x)\right) u(x)\right\rangle d x+\int_{\Omega} V(x)|u(x)|^{2} d x \\
= & \int_{\Omega_{\Psi}}\left\langle\omega^{2}(y)\left(-i \nabla_{y}-A_{*}(y)\right) \psi_{*}(y) v(y),\left(-i \nabla_{y}-A_{*}(y)\right) \psi_{*}(y) v(y)\right\rangle d y \\
& \quad+\int_{\Omega_{\Psi}} V_{*}(y) \psi_{*}(y)^{2}|v(y)|^{2} d y \\
= & \int_{\Omega_{*}}\left\langle\omega^{2}(y)\left(-i \nabla_{y}-A_{*}(y)\right) \psi_{*}(y) v(y),\left(-i \nabla_{y}-A_{*}(y)\right) \psi_{*}(y) v(y)\right\rangle d y \\
& \quad+\int_{\Omega_{*}} V_{*}(y) \psi_{*}(y)^{2}|v(y)|^{2} d y \\
= & \left(H\left(0 ; \omega^{2} \mathbf{1}, A_{*}, V_{*}\right) \psi_{*} v, \psi_{*} v\right)_{\mathrm{L}^{2}\left(\Omega_{*}\right)} .
\end{aligned}
$$

THEOREM 6.3. Suppose that $k \in \mathbb{R}^{2}$. Under the assumptions of Theorem 2.1, the operator $H(k ; g, A, V)$ is unitarily equivalent to the operator

$$
H\left(\left(T_{*}^{-1}\right)^{t} k, \omega^{2} \psi_{*}^{2} \mathbf{1}, A_{*}, \psi_{*}^{2} V_{*}+\psi_{*}^{2} \omega \Delta \omega-\psi_{*} \omega \Delta\left(\psi_{*} \omega\right)\right)
$$


acting in $\mathrm{L}^{2}\left(\Omega_{*}\right)$, where $\Omega_{*} \subset \mathbb{R}^{2}$ is an elementary cell of $\Gamma_{*}$, and $T_{*}, \omega, \psi_{*}, A_{*}$ and $V_{*}$ are defined above.

Proof. We will perform the required unitary transformation in several steps. First, let us note that $H(k ; g, A, V)=H(0 ; g, A-k, V)$. Consider the unitary transformation $u(x)=e^{i \alpha(x)} v(x)$, where $\alpha \in \mathrm{C}_{\text {per }}^{1}(\Omega)$. Note that, under this transformation, the operator $H(k ; g, A, V)$ becomes $H(0 ; g, A-k-\nabla \alpha, V)$. Take $\alpha(x)=k\left(T_{*}^{-1} \Psi(x)-x\right)$. This function is $\Gamma$-periodic, and

$$
(\nabla \alpha)(x)=\Psi^{\prime}(x)^{t}\left(T_{*}^{-1}\right)^{t} k-k .
$$

Hence, the operator $H(k ; g, A, V)$ is unitarily equivalent to $H\left(0, g, A-\Psi^{\prime}(x)^{t}\left(T_{*}^{-1}\right)^{t} k, V\right)$, which, by Lemma 6.2 , is equivalent to

$$
\psi_{*} H\left(0, \omega^{2} \mathbf{1}, A_{*}-\left(T_{*}^{-1}\right)^{t} k, V_{*}\right) \psi_{*}=\psi_{*} H\left(\left(T_{*}^{-1}\right)^{t} k, \omega^{2} \mathbf{1}, A_{*}, V_{*}\right) \psi_{*} .
$$

Applying (5.3) and then (5.4), we ultimately obtain

$$
\begin{array}{rl}
\psi_{*} & H\left(\left(T_{*}^{-1}\right)^{t} k, \omega^{2} \mathbf{1}, A_{*}, V_{*}\right) \psi_{*} \\
& =\omega \psi_{*} H\left(\left(T_{*}^{-1}\right)^{t} k, \mathbf{1}, A_{*}, \omega^{-2} V_{*}+\omega^{-1} \Delta \omega\right) \omega \psi_{*} \\
& =H\left(\left(T_{*}^{-1}\right)^{t} k, \omega^{2} \psi_{*}^{2} \mathbf{1}, A_{*}, \psi_{*}^{2} V_{*}+\psi_{*}^{2} \omega \Delta \omega-\psi_{*} \omega \Delta\left(\psi_{*} \omega\right)\right) .
\end{array}
$$

This completes the proof of Theorem 2.1, because its statement has already been established for the operators (6.2), and the operator families $H(k ; g, A, V)$ and (6.2) have the same band functions up to a linear transformation of $k$.

\section{An example of degenerate band edge in the discrete case}

Consider the discrete Schrödinger operator $H=\mathcal{D}+V$ in $l^{2}\left(\mathbb{Z}^{2}\right)$, where

$$
(\mathcal{D} u)_{n}=\frac{1}{2}\left(u_{n+e_{1}}+u_{n-e_{1}}+u_{n+e_{2}}+u_{n-e_{2}}\right), \quad n \in \mathbb{Z}^{2},
$$

is the discrete Laplace operator, and $V$ is the operator of multiplication by the potential given by

$$
(V u)_{n}= \begin{cases}v_{0} u_{n}, & \text { if } n_{1}+n_{2} \text { is even, } \\ v_{1} u_{n}, & \text { if } n_{1}+n_{2} \text { is odd, }\end{cases}
$$

where the real numbers $v_{0}$ and $v_{1}$ are fixed. In other words, the lattice is formed by two different types of atoms placed in a chessboard order, and $V$ is periodic with respect to the lattice spanned by $\left\{2 e_{1}, e_{1}+e_{2}\right\}$. The corresponding Floquet-Bloch transform

$$
F: l^{2}\left(\mathbb{Z}^{2}\right) \longrightarrow \mathrm{L}^{2}(\widetilde{\mathcal{O}} \times\{0 ; 1\})
$$


is given by

$$
(F u)(k ; m)=\frac{1}{\pi \sqrt{2}} \sum_{n_{1}+n_{2} \equiv m(\bmod 2)} e^{-i k n} u_{n} .
$$

Here $k \in \widetilde{\mathcal{O}}=\left\{k \in \mathbb{R}^{2}:\left|k_{1}+k_{2}\right|<\pi\right\}$, and $m \in\{0,1\}$. The operator $F$ is unitary, and it is easy to see that

$$
F H F^{*}=\int_{\widetilde{\mathcal{O}}}^{\oplus} H(k) d k
$$

where $H(k)$ is a self-adjoint operator in $\mathbb{C}^{2}$,

$$
H(k)=\left(\begin{array}{cc}
v_{0} & \cos k_{1}+\cos k_{2} \\
\cos k_{1}+\cos k_{2} & v_{1}
\end{array}\right) .
$$

Eigenvalues of this matrix are

$$
\lambda_{ \pm}(k)=\frac{v_{0}+v_{1}}{2} \pm \sqrt{\left(\frac{v_{0}-v_{1}}{2}\right)^{2}+\left(\cos k_{1}+\cos k_{2}\right)^{2}}
$$

from which it follows that

$$
\begin{array}{ll}
\min \lambda^{-}=\frac{v_{0}+v_{1}}{2}-\sqrt{\left(\frac{v_{0}-v_{1}}{2}\right)^{2}+4}, & \max \lambda^{-}=\min \left(v_{0}, v_{1}\right) \\
\min \lambda^{+}=\max \left(v_{0}, v_{1}\right), & \max \lambda^{+}=\frac{v_{0}+v_{1}}{2}+\sqrt{\left(\frac{v_{0}-v_{1}}{2}\right)^{2}+4}
\end{array}
$$

So, the spectrum of the operator $H$ consists of two bands separated by a gap, whenever $v_{0} \neq v_{1}$.

The edges of this gap ( $v_{0}$ and $v_{1}$, respectively) are attained on the set

$$
\left\{k \in \mathbb{R}^{2}: \cos k_{1}+\cos k_{2}=0\right\}=\left\{k \in \mathbb{R}^{2}: k_{1} \pm k_{2}=(2 p+1) \pi\right\}_{p \in \mathbb{Z}},
$$

which is a countable union of straight lines. Figure 2 shows the graphs of $\lambda_{ \pm}(\cdot)$ for $v_{0}=0$, $v_{1}=2$, with the dashed lines indicating the level sets at the edges of the gap $[0,2]$.

Remark 7.1. This example seems to be one of the simplest possible 2-dimensional diatomic tight binding models. We believe that it should be known to the experts in solid state physics. We could not, however, find it in the literature, which is the reason why we discuss it in detail. 


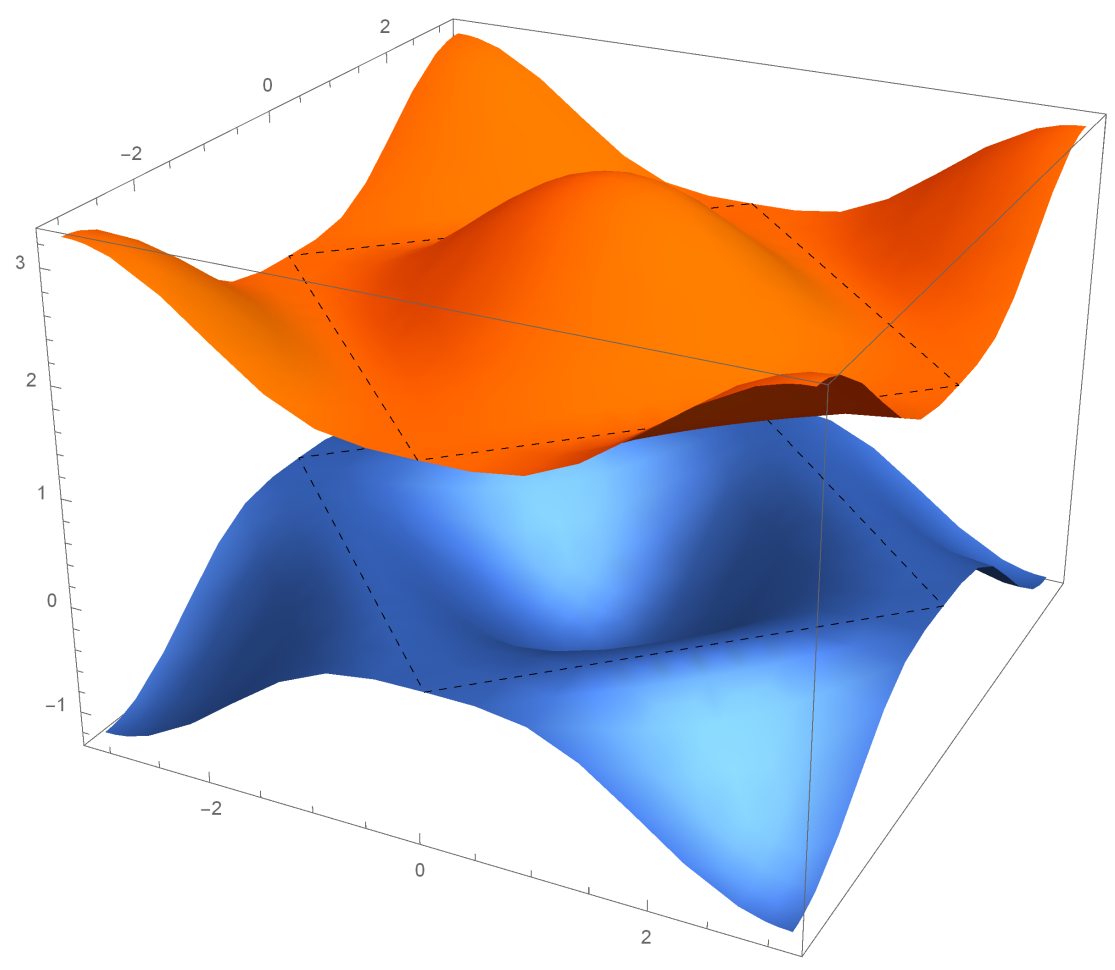

Figure 2. The band functions $\lambda^{+}(k)$ and $\lambda^{-}(k)$.

\section{References}

[1] Ashcroft, N. W. \& Mermin, N. D., Solid State Physics. Brooks/Cole, Belmont, CA, 1976.

[2] Birman, M. Sh. \& Suslina, T. A., The two-dimensional periodic magnetic Hamiltonian is absolutely continuous. Algebra i Analiz, 9 (1997), 32-48 (Russian); English translation in St. Petersburg Math. J., 9 (1998), 21-32.

[3] - Absolute continuity of a two-dimensional periodic magnetic Hamiltonian with discontinuous vector potential. Algebra i Analiz, 10 (1998), 1-36 (Russian); English translation in St. Petersburg Math. J., 10 (1999), 579-601.

[4] - A periodic magnetic Hamiltonian with a variable metric. The problem of absolute continuity. Algebra i Analiz, 11 (1999), 1-40 (Russian); English translation in St. Petersburg Math. J., 11 (2000), 203-232.

[5] - Two-dimensional periodic Pauli operator. The effective masses at the lower edge of the spectrum, in Mathematical Results in Quantum Mechanics (Prague, 1998), Oper. Theory Adv. Appl., 108, pp. 13-31. Birkhäuser, Basel, 1999.

[6] - Periodic second-order differential operators. Threshold properties and averaging. Algebra i Analiz, 15 (2003), 1-108 (Russian); English translation St. Petersburg Math. J., 15 (2004), 639-714. 
[7] Hoang, V. \& Radosz, M., Absence of bound states for waveguides in two-dimensional periodic structures. J. Math. Phys., 55 (2014), 033506, 20 pp.

[8] Kato, T., Perturbation Theory for Linear Operators. Classics in Mathematics. Springer, Berlin-Heidelberg, 1995.

[9] KHA, M., Green's function asymptotics of periodic elliptic operators on abelian coverings of compact manifolds. J. Funct. Anal., 274 (2018), 341-387.

[10] Kha, M., Kuchment, P. \& Raich, A., Green's function asymptotics near the internal edges of spectra of periodic elliptic operators. Spectral gap interior. J. Spectr. Theory, 7 (2017), 1171-1233.

[11] Kirsch, W. \& Simon, B., Comparison theorems for the gap of Schrödinger operators. J. Funct. Anal., 75 (1987), 396-410.

[12] Klopp, F. \& RAlston, J., Endpoints of the spectrum of periodic operators are generically simple. Methods Appl. Anal., 7 (2000), 459-463.

[13] Kuchment, P., Floquet Theory for Partial Differential Equations. Operator Theory: Advances and Applications, 60. Birkhäuser, Basel, 1993.

[14] - The mathematics of photonic crystals, in Mathematical Modeling in Optical Science, Frontiers Appl. Math., 22, pp. 207-272. SIAM, Philadelphia, PA, 2001.

[15] - An overview of periodic elliptic operators. Bull. Amer. Math. Soc., 53 (2016), 343-414.

[16] Kuchment, P. \& Levendorskî̂, S., On the structure of spectra of periodic elliptic operators. Trans. Amer. Math. Soc., 354 (2002), 537-569.

[17] Kuchment, P. \& Pinchover, Y., Integral representations and Liouville theorems for solutions of periodic elliptic equations. J. Funct. Anal., 181 (2001), 402-446.

[18] - Liouville theorems and spectral edge behavior on abelian coverings of compact manifolds. Trans. Amer. Math. Soc., 359 (2007), 5777-5815.

[19] Kuchment, P. \& Raich, A., Green's function asymptotics near the internal edges of spectra of periodic elliptic operators. Spectral edge case. Math. Nachr., 285 (2012), $1880-1894$.

[20] Parnovski, L. \& Shterenberg, R., Perturbation theory for spectral gap edges of 2D periodic Schrödinger operators. J. Funct. Anal., 273 (2017), 444-470.

[21] Reed, M. \& Simon, B., Methods of Modern Mathematical Physics. IV. Analysis of Operators. Academic Press, New York-London, 1978.

[22] Shterenberg, R. G., Absolute continuity of the spectrum of the two-dimensional periodic Schrödinger operator with strongly subordinate magnetic potential. Zap. Nauchn. Sem. S.-Peterburg. Otdel. Mat. Inst. Steklov. (POMI), 303 (2003), 279-320 (Russian); English translation in J. Math. Sci. (N.Y.), 129 (2005), 4087-4109.

[23] — An example of a periodic magnetic Schrödinger operator with a degenerate lower edge of the spectrum. Algebra i Analiz, 16 (2004), 177-185 (Russian); English translation in St. Petersburg Math. J., 16 (2005), 417-422.

[24] - On the structure of the lower edge of the spectrum of a periodic magnetic Schrödinger operator with small magnetic potential. Algebra i Analiz, 17 (2005), 232-243 (Russian); English translation in St. Petersburg Math. J., 17 (2006), 865-873.

[25] Colin de Verdière, Y., Sur les singularités de van Hove génériques. Mém. Soc. Math. France, 46 (1991), 99-110.

[26] VAn der Waerden, B. L., Algebra. Vol. II. Springer, New York, 1991.

[27] Zắdenberg, M. G., Krĕ̌n, S. G., Kuchment, P. A. \& Pankov, A. A., Banach bundles and linear operators. Uspehi Mat. Nauk, 30 (1975), 101-157 (Russian); English translation in Russian Math. Surveys, 30 (1975), 115-175. 
Nikolay Filonov

St. Petersburg Department

V. A. Steklov Mathematical Institute

Fontanka 27,

St. Petersburg, 191023

Russia

and

St. Petersburg State University

Universitetskaya emb. 7/9

St. Petersburg, 199034

Russia

filonov@pdmi.ras.ru

Received March 26, 2016
ILYA KaChKOVSKIY

Department of Mathematics

Michigan State University

Wells Hall, 619 Red Cedar Road

East Lansing, MI, 48910

U.S.A.

ikachkov@msu.edu 